\title{
Effects of late exercise on cardiac remodeling and myocardial calcium handling proteins in rats with moderate and large size myocardial infarction is
}

\author{
Daniele M. Guizoni ${ }^{\text {a }}$, Silvio A. Oliveira-Junior ${ }^{\mathrm{b}}$, Sefora L.R. Noor ${ }^{\mathrm{a}}$, Luana U. Pagan ${ }^{\mathrm{a}}$, Paula F. Martinez ${ }^{\mathrm{a}, \mathrm{b}}$, \\ Aline R.R. Lima ${ }^{a}$, Mariana J. Gomes ${ }^{\text {a }}$, Ricardo L. Damatto ${ }^{a}$, Marcelo D.M. Cezar ${ }^{\text {a }}$, Camila Bonomo ${ }^{\text {a }}$, \\ Leonardo A.M. Zornoff ${ }^{\text {a }}$, Katashi Okoshi ${ }^{\text {, }}$, Marina P. Okoshi ${ }^{\text {a,* }}$ \\ a Internal Medicine Department, Botucatu Medical School, Sao Paulo State University, UNESP, Botucatu, Brazil \\ ${ }^{\mathrm{b}}$ School of Physical Therapy, Federal University of Mato Grosso do Sul, Campo Grande, Brazil
}

\section{A R T I C L E I N F O}

\section{Article history:}

Received 13 May 2016

Accepted 4 July 2016

Available online 5 July 2016

\section{Keywords:}

Myocardial infarction

Physical exercise

Cardiac function

Echocardiogram

Calsequestrin

Remodeling

\begin{abstract}
A B S T R A C T
Background: Physical exercise attenuates myocardial infarction (MI)-induced cardiac remodeling. However, it is unsettled whether late exercise modulates post-infarction cardiac remodeling differentially according to infarct size. We investigated the effects of exercise started at late stage heart failure on cardiac remodeling in rats with moderate and large sized MI.

Methods: Three months after MI, rats were assigned into sedentary and exercise groups. Exercise rats underwent treadmill for three months. After assessing infarct size by histological analysis, rats were subdivided into four groups: moderate MI sedentary (Mod MI-Sed; $n=7$ ), Mod MI exercised (Mod MI-Ex; $n=7$ ), Large MI-Sed $(\mathrm{n}=11)$, and Large MI-Ex $(\mathrm{n}=10)$.

Results: Before exercise, MI-induced cardiac changes were demonstrated by comparing results to a Sham group; alterations were more intense in rats with large than moderate MI size. Systolic function, evaluated by echocardiogram using the variation in LV fractional area change between after and before exercise, was improved in exercise than sedentary groups. Calsequestrin expression increased in exercised compared to sedentary groups. L-type calcium channel was higher in Mod MI-Ex than Mod MI-Sed. SERCA2a, phospholamban, and $\mathrm{Na}^{+} / \mathrm{Ca}^{2+}$ exchanger expression did not differ between groups.

Conclusion: Late exercise improves systolic function and modulates intracellular calcium signaling proteins in rats with moderate and large MI.
\end{abstract}

(c) 2016 Elsevier Ireland Ltd. All rights reserved.

\section{Introduction}

Heart failure is a major worldwide cause of disability and death [1]. Animal myocardial infarction (MI) models are relevant in pathophysiology and treatment studies as myocardial ischemia and infarction are common causes of cardiac remodeling and heart failure in humans [1]. After MI, cardiac remodeling is associated with progressive ventricular dysfunction, decompensated heart failure, and cardiovascular death. Despite progressive improvement in pharmacological treatment, heart failure prognosis is still poor [2]. Efforts have therefore been directed towards non-pharmacological treatment, and physical exercise is

\footnotetext{
it All authors have read and approved the manuscript submission.

* Corresponding author at: Departamento de Clinica Medica, Faculdade de Medicina de Botucatu, UNESP, Rubiao Junior, S/N 18618-970 Botucatu, SP, Brazil.

E-mail address: mpoliti@fmb.unesp.br (M.P. Okoshi).
}

recommended as an important strategy in attenuating post-infarction cardiac remodeling and heart failure development [2].

Ligation of the left anterior descending coronary artery in rats leads to a wide range of infarction size, which modulates the ensuing cardiac remodeling and left ventricular (LV) dysfunction [3,4]. The effects of physical exercise have been assessed in rats with different myocardial infarction sizes, ranging from $30 \%$ to $40 \%$ [5-10] and over $40 \%$ total LV area [6,9,11-14]. Aerobic exercise in rats with moderate size myocardial infarct has been shown to improve survival, systolic and mitochondrial function, and inflammatory profile [5,7-9]. Furthermore, high-intensity sprint training [10] attenuated myocyte hypertrophy and normalized $\mathrm{Ca}^{2+}$ transient and sarcoplasmic reticulum function in myocytes. Exercise in rats with large MI improved cardiac remodeling and ventricular function, which was associated with restored contractile function and intracellular $\mathrm{Ca}^{2+}$ handling, and decreased myocardial fibrosis and oxidative stress [11-14]. In contrast, swim training in rats with large MI decreased survival and impaired LV remodeling [6]. Only a 


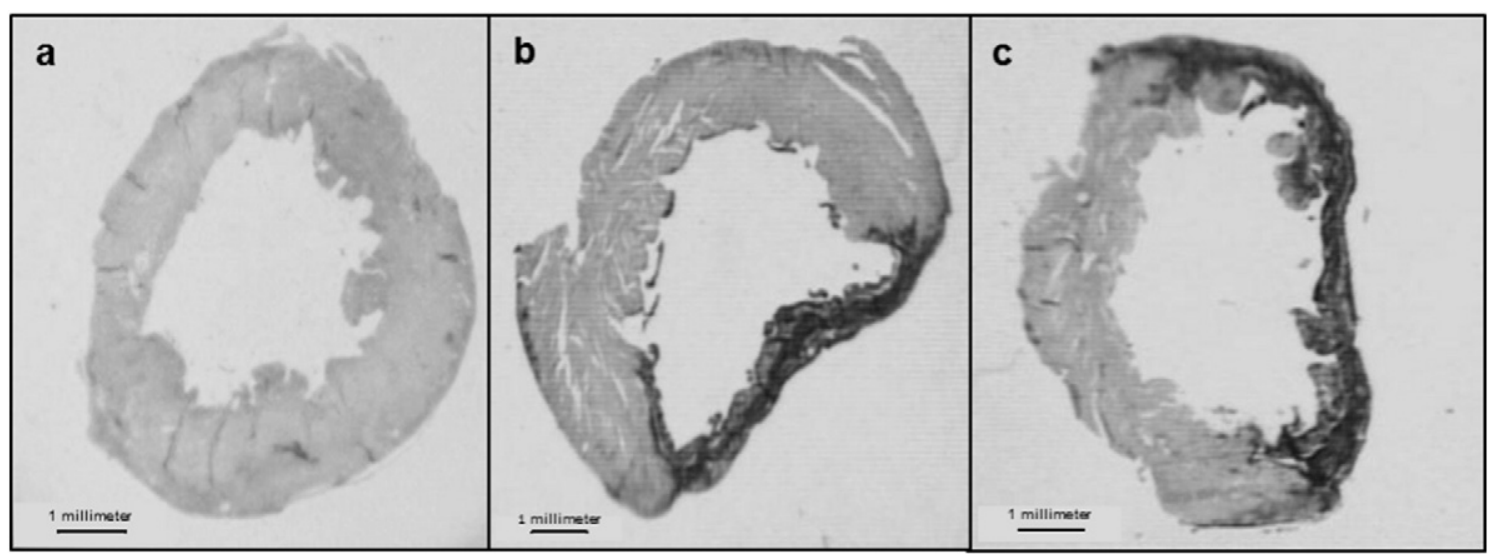

Fig. 1. Representative histological photos of Sirius red F3BA-stained left ventricles from Sham (A), and infarcted rats with moderate (B) and large (C) myocardial infarction sizes.

few authors have examined the effects of aerobic exercise on late stage cardiac remodeling after MI induction, when heart failure is more prevalent. At this stage, beneficial [15] effects were observed in cardiac remodeling.

It is therefore still not clear whether late exercise modulates post-infarction cardiac remodeling differentially according to infarct size. In this study we investigated the effects of a treadmill exercise protocol started at a late stage heart failure on cardiac remodeling and myocardial calcium handling protein expression in rats with moderate and large size myocardial infarction.

\section{Materials and methods}

\subsection{Experimental design}

Male Wistar rats (200-250 g, 50-60 days old) were purchased from the Central Animal House, Botucatu Medical School, UNESP. All animals were housed in a room under temperature control at $24{ }^{\circ} \mathrm{C}$ and kept on a 12-hour light/dark cycle. Food and water were supplied ad libitum. All experiments and procedures were performed in accordance with the Canadian Council on Animal Care guidelines and were approved by Botucatu Medical School Ethics Committee, UNESP, SP, Brazil (protocol number 808).

MI was induced according to a previously described method $(n=60)[16,17]$. Briefly rats were anesthetized with ketamine $(70 \mathrm{mg} / \mathrm{kg})$ and xylazine $(1 \mathrm{mg} / \mathrm{kg})$ and subjected to left lateral thoracotomy. After heart exteriorization, the left atrium was retracted to facilitate ligation of the left anterior descending coronary artery with 5-0 mononylon suture between the pulmonary outflow tract and the left atrium. The heart was then replaced into the thorax, the lungs were inflated with positive pressure, and the thoracotomy was closed. To demonstrate infarction-induced cardiac injury before the exercise protocol, results of infarcted rats were compared with those from sham-operated rats $(n=12)$

Table 1

Cardiac structural parameters analyzed by transthoracic echocardiogram before exercise protocol.

\begin{tabular}{lrcc}
\hline & \multicolumn{1}{l}{$\begin{array}{l}\text { Sham } \\
(\mathrm{n}=12)\end{array}$} & $\begin{array}{l}\text { Moderate MI } \\
(\mathrm{n}=15)\end{array}$ & $\begin{array}{l}\text { Large MI } \\
(\mathrm{n}=29)\end{array}$ \\
\hline LVDD, mm & $8.38 \pm 0.35$ & $10.25 \pm 0.62^{*}$ & $10.78 \pm 0.70^{*}, * *$ \\
LVDD/BW, mm/kg & $18.78 \pm 1.73$ & $22.99 \pm 1.79^{*}$ & $22.75 \pm 1.87^{*}$ \\
LVSD, mm & $4.05 \pm 0.44$ & $7.72 \pm 0.97^{*}$ & $8.57 \pm 0.83^{*}, * *$ \\
PWT, mm & $1.35 \pm 0.07$ & $1.53 \pm 0.12^{*}$ & $1.60 \pm 0.16^{*}$ \\
AO, mm & $3.70 \pm 0.26$ & $3.63 \pm 0.22$ & $3.59 \pm 0.24$ \\
LA, mm & $5.55 \pm 0.54$ & $6.73 \pm 0.65^{*}$ & $7.69 \pm 0.66^{*, * *}$ \\
LA/AO & $1.50 \pm 0.16$ & $1.84 \pm 0.17^{*}$ & $2.10 \pm 0.53^{*}$ \\
LA/BW, mm/kg & $12.43 \pm 1.44$ & $15.19 \pm 1.83^{*}$ & $16.06 \pm 1.64^{*}$ \\
LV diastolic area, $\mathrm{cm}^{2}$ & $0.52 \pm 0.07$ & $0.79 \pm 0.11^{*}$ & $0.81 \pm 0.07^{*, * *}$ \\
LV systolic area, $\mathrm{cm}^{2}$ & $0.16 \pm 0.03$ & $0.54 \pm 0.07^{*}$ & $0.63 \pm 0.08^{*}, * *$ \\
\hline
\end{tabular}

Data are expressed as mean \pm SD. Moderate MI: rats with myocardial infarction (MI) size $<40 \%$ total left ventricle (LV) endocardial and epicardial circumferences; Large MI: rats with MI size $\geq 40 \%$ total LV circumferences; LVDD and LVSD: LV diastolic and systolic diameters, respectively; PWT: LV diastolic posterior wall thickness; AO: aorta diameter; LA: left atrium diameter; BW: body weight. ANOVA and Student Newman-Keuls test.

* $P<0.05$ vs Sham.

** $P<0.05$ vs Moderate MI
Three months after surgery, survival rats were subjected to echocardiographic evaluation and assigned into sedentary $(n=22)$ and exercised groups $(n=22)$. Physical exercise protocol consisted of $40 \mathrm{~min} /$ day treadmill running at $16 \mathrm{~m} / \mathrm{min}, 5$ days/week, for three months [15]. During an adaptive period of two weeks, exercise velocity and duration were slowly increased. In the first two weeks of training, the animals were subjected to low-voltage electrical stimulation to start exercise. At the end of the exercise protocol, rats were subjected to echocardiographic evaluation and euthanized the next day for tissue collection. After assessment of myocardial infarction size by histological analyses, rats were then subdivided into four groups: sedentary rats with moderate MI size (Mod IM-Sed, $\mathrm{n}=7$ ), sedentary rats with large MI (Large MI-Sed, $\mathrm{n}=11$ ), exercised rats with moderate MI (Mod MI-Ex, $\mathrm{n}=7$ ) and exercised rats with large MI (Large MI-Ex, $\mathrm{n}=10$ ). Moderate MI groups contained rats with MI size $<40 \%$ sum of total (infarcted and viable myocardium) endocardial and epicardial ventricular circumferences, and large MI groups contained rats with MI size $\geq 40 \%$ total LV circumferences [18].

\subsection{Echocardiographic study}

Echocardiography was performed using a commercially available echocardiograph (General Electric Medical Systems, Vivid S6, Tirat Carmel, Israel) equipped with a 511.5 $\mathrm{MHz}$ multifrequency probe. Rats were anesthetized by intramuscular injection with a mixture of ketamine $(50 \mathrm{mg} / \mathrm{kg})$ and xylazine $(0.5 \mathrm{mg} / \mathrm{kg})$. A two-dimensional parasternal short-axis view of the LV was obtained at the level of the papillary muscles. M-mode tracings were obtained from short-axis views of the LV at or just below the tip of the mitral-valve leaflets, and at the level of the aortic valve and left atrium [19-21]. M-mode images of the LV were printed on a black-and-white thermal printer (Sony UP$890 \mathrm{MD}$ ) at a sweep speed of $100 \mathrm{~mm} / \mathrm{s}$. All LV structures were manually measured by the same observer (KO), who was blind to the experimental groups. Measurements were the mean of at least five cardiac cycles on M-mode tracings. The following structural variables were evaluated: left atrium (LA) diameter, aorta diameter (AO), LV diastolic and systolic diameters (LVDD and LVSD, respectively), LV diastolic posterior wall thickness (PWT), and LV diastolic and systolic area. LV function was assessed by the following parameters: posterior wall shortening velocity (PWSV), fractional area change (FAC), early

Table 2

Left ventricular functional parameters analyzed by transthoracic echocardiogram before exercise protocol.

\begin{tabular}{llcc}
\hline & $\begin{array}{l}\text { Sham } \\
(\mathrm{n}=12)\end{array}$ & $\begin{array}{l}\text { Moderate MI } \\
(\mathrm{n}=15)\end{array}$ & $\begin{array}{l}\text { Large MI } \\
(\mathrm{n}=29)\end{array}$ \\
\hline HR, Beats/min & $262 \pm 18$ & $301 \pm 45^{*}$ & $297 \pm 38^{*}$ \\
PWSV, mm/s & $35.6 \pm 4.50$ & $25.3 \pm 5.95^{*}$ & $22.8 \pm 5.94^{*}$ \\
FAC, \% & $69.6 \pm 3.84$ & $32.2 \pm 6.93^{*}$ & $29.2 \pm 5.85^{*}$ \\
E-wave, cm/s & $84.5 \pm 8.78$ & $97.0 \pm 17.21$ & $108.1 \pm 21.44^{*}$ \\
A wave, cm/s & $49.1 \pm 11.98$ & $67.1 \pm 26.49^{*}$ & $37.3 \pm 18.19^{* *}$ \\
E/A & $1.80 \pm 0.40$ & $1.90 \pm 1.55$ & $3.82 \pm 2.19^{*}, * *$ \\
IVRT, ms & $26.2 \pm 4.79$ & $33.0 \pm 6.21^{*}$ & $26.9 \pm 6.12^{* *}$ \\
EDT, ms & $48.2 \pm 5.84$ & $44.3 \pm 12.52$ & $37.1 \pm 5.57^{*}, * *$ \\
\hline
\end{tabular}

Data expressed as mean \pm SD. Moderate MI: rats with myocardial infarction (MI) size $<40 \%$ total left ventricle (LV) endocardial and epicardial circumferences; Large MI: rats with MI size $\geq 40 \%$ total LV circumferences; HR, heart rate; PWSV: posterior wall shortening velocity; FAC: fractional area change; E/A: early-to-late diastolic mitral inflow ratio; IVRT: isovolumetric relaxation time; EDT: E-wave deceleration time. ANOVA and Student Newman-Keuls test.

* $P<0.05$ vs Sham.

** $P<0.05$ vs Moderate MI. 
Table 3

Cardiac structural parameters analyzed by transthoracic echocardiogram after exercise protocol.

\begin{tabular}{lllll}
\hline & $\begin{array}{l}\text { Mod } \\
\text { MI-Sed } \\
(\mathrm{n}=7)\end{array}$ & $\begin{array}{l}\text { Mod MI-Ex } \\
(\mathrm{n}=7)\end{array}$ & $\begin{array}{l}\text { Large MI-Sed } \\
(\mathrm{n}=11)\end{array}$ & $\begin{array}{l}\text { Large MI-Ex } \\
(\mathrm{n}=10)\end{array}$ \\
\hline LVDD, mm & $9.85 \pm 0.90$ & $10.68 \pm 0.91$ & $11.81 \pm 1.02^{*}$ & $11.52 \pm 0.90$ \\
LVDD/BW, mm/kg & $20.8 \pm 3.7$ & $21.4 \pm 1.7$ & $22.1 \pm 2.4^{*}$ & $22.8 \pm 1.8$ \\
LVSD, mm & $6.65 \pm 1.37$ & $8.18 \pm 1.08$ & $9.87 \pm 1.41$ & $9.30 \pm 1.17$ \\
PWT, mm & $1.43 \pm 0.18$ & $1.70 \pm 0.28$ & $1.62 \pm 0.26$ & $1.59 \pm 0.30$ \\
AO, mm & $3.77 \pm 0.18$ & $3.79 \pm 0.21$ & $3.77 \pm 0.19$ & $3.77 \pm 0.18$ \\
LA, mm & $6.59 \pm 1.16$ & $7.33 \pm 1.00$ & $8.67 \pm 1.44^{*}$ & $8.26 \pm 0.93$ \\
LA/AO & $1.75 \pm 0.30$ & $1.94 \pm 0.27$ & $2.30 \pm 0.40^{*}$ & $2.20 \pm 0.32$ \\
LA/BW, mm/kg & $14.0 \pm 3.4$ & $14.7 \pm 2.0$ & $16.3 \pm 3.0$ & $16.3 \pm 1.7$ \\
LV diastolic area, & $0.83 \pm 0.09$ & $0.86 \pm 0.17$ & $1.06 \pm 0.16^{*}$ & $1.00 \pm 0.17$ \\
$\quad$ cm $^{2}$ & & & & \\
LV systolic area, $\mathrm{cm}^{2}$ & $0.57 \pm 0.03$ & $0.55 \pm 0.11$ & $0.82 \pm 0.15^{*}$ & $0.69 \pm 0.12^{* *}$ \\
\hline
\end{tabular}

Data expressed as mean \pm SD. Mod MI-Sed: sedentary rats with myocardial infarction (MI) size $<40 \%$ total left ventricle (LV) endocardial and epicardial circumferences; Mod MI-Ex: exercised rats with MI size $<40 \%$ total LV circumferences; Large MI-Sed: sedentary rats with MI size $\geq 40 \%$ total LV circumferences; Large MI-Ex: exercised rats with MI size $\geq 40 \%$ total LV circumferences; LVDD and LVSD: LV diastolic and systolic diameters, respectively; BW: body weight; PWT: LV diastolic posterior wall thickness; AO: aorta diameter; LA: left atrium diameter. ANOVA and Student Newman-Keuls test.

* $P<0.05$ vs Mod MI-Sed.

** $P<0.05$ vs Large MI-Sed.

and late diastolic mitral inflow velocities ( $\mathrm{E}$ and $\mathrm{A}$ waves), E/A ratio, isovolumetric relaxation time (IVRT), and E-wave deceleration time (EDT). Heart rate (HR) was calculated using an average of five consecutive R-R intervals.

\subsection{Morphological studies}

After anesthesia with sodium pentobarbital $(50 \mathrm{mg} / \mathrm{kg})$, rats were euthanized by decapitation. The heart was removed by thoracotomy and the atria and ventricles were separated and weighed. For infarct size measurement, transverse LV samples were fixed in a $10 \%$ buffered formalin solution for $48 \mathrm{~h}$ and embedded in paraffin. Five-micrometerthick sections were cut from the blocked tissued and stained with hematoxylin-eosin and with the collagen-specific stain picrosirius red (Sirius red F3BA in aqueous saturated picric acid) as previously described [22].

The lengths of infarcted and viable myocardial for both endocardial and epicardial circumferences were determined by planimetry in hearts stained with picro-sirius red. Infarction size was calculated by dividing the sum of endocardial and epicardial infarcted ventricular lengths by the sum of total (infarcted and viable myocardium) endocardial and epicardial ventricular circumferences. The measurements were taken from midventricular slices (5-6 mm from the apex), assuming that the left midventricular slice shows a close linear relationship with the sum of measurements from all heart slices [23].

Cardiomyocyte diameters were measured as the shortest distance between borders drawn across the nucleus and averaged from 5 to 10 different fields of the LV posterior wall (50-100 cardiomyocytes/heart) in hematoxylin and eosin stained sections from each heart $[24,25]$. Measurements were taken using a compound microscope (Leica DM LS; Nussloch, Germany) attached to a computerized imaging analysis system (Media Cybernetics, Silver Spring, MD).

\subsection{Western blotting analysis}

LV myocardial protein levels were analyzed by Western blot as previously described [26-28] using specific antibodies to detect sarcoendoplasmic reticulum $\mathrm{Ca}^{2+}$-ATPase (SERCA)2a (IID8, Thermo Scientific), phospholamban (2D12, Thermo Scientific), calsequestrin (H-60, sc-28275, Santa Cruz Biotechnology), voltage-sensitive L-type Ca ${ }^{2+}$ channel (H-280, sc 25686, Santa Cruz Biotechnology), and $\mathrm{Na}^{+} / \mathrm{Ca}^{2+}$ exchanger ( $22 \mathrm{C12}$, Thermo Scientific). Protein levels were normalized to GAPDH (6C5, sc-32233, Santa Cruz Biotechnology). Muscle protein was extracted using Tris-Triton buffer (10 mM Tris pH 7.4, 100 mM NaCl, 1 mM EDTA, 1 mM EGTA, 1\% Triton X-100, 10\% glycerol, 0.1\% SDS, $0.5 \%$ deoxycholate). Supernatant protein content was quantified by Bradford's method. Samples were separated on polyacrylamide gel and transferred to a nitrocellulose membrane. After blockage, the membrane was incubated with primary antibodies. The membrane was then washed with TBS and Tween 20 and incubated with secondary peroxidase-conjugated antibodies. Super Signal ${ }^{\circledR}$ West Pico Chemiluminescent Substrate (Pierce Protein Research Products, Rockford, USA) was used to detect bound antibodies.

\subsection{Statistical analysis}

Data are expressed as mean \pm standard deviation. Comparisons among Sham and infarcted groups were performed by one-way ANOVA followed by the Student NewmanKeuls test. Comparisons among infarcted groups were performed by two-way ANOVA followed by the Student Newman-Keuls test. Level of significance was considered at $5 \%$.

\section{Results}

Mortality after MI induction and before exercise protocol was 27\%. During exercise period, mortality was as follows: Mod MI-Sed absent; Mod MI-Ex 12.5\% (1 rat); Large MI-Sed 26.6\% (4 rats); Large MI-Ex 28.5\% (4 rats). No animals were lost during exercise performance.

Experimental groups were divided into moderate and large MI sizes after post-mortem histological assessment of infarction size. MI size was larger in both large MI groups than moderate MI groups (Mod MI-Sed $34.9 \pm 3.3$; Mod MI-Ex $34.8 \pm 4.5$; Large MI-Sed $49.6 \pm 5.1$; Large MI-Ex $47.4 \pm 3.8 \%$ of total LV area; $P<0.05$; Fig. 1 ). MI size did not significantly differ between exercise and sedentary groups.

Infarction-induced cardiac injury was characterized by subjecting rats to transthoracic echocardiographic evaluation before the exercise protocol and results compared to a Sham-operated group. No differences were observed between exercised and sedentary groups according to the infarction size (data not shown). Rats with moderate and large MI presented increased LV systolic and diastolic diameters, left atrial diameter, LV posterior wall thickness (PWT), and LV diastolic and systolic areas compared to Sham group (Table 1). Large MI group had higher LV systolic and diastolic diameters, left atrial diameter, and LV systolic and diastolic areas than the Moderate MI group. Table 2 shows LV functional parameters. Moderate and Large MI groups presented increased heart rate, and reduced LV posterior wall shortening velocity and LV fractional area change than Sham. The Large MI group had a lower A wave, isovolumetric relaxation time, and E-wave deceleration time, and increased $\mathrm{E} / \mathrm{A}$ ratio than the Moderate MI group.

Table 4

Left ventricular functional parameters analyzed by transthoracic echocardiogram after exercise protocol.

\begin{tabular}{|c|c|c|c|c|}
\hline & Mod MI-Sed $(\mathrm{n}=7)$ & Mod MI-Ex $(\mathrm{n}=7)$ & Large MI-Sed $(\mathrm{n}=11)$ & Large MI-Ex $(\mathrm{n}=10)$ \\
\hline HR, beats/min & $287 \pm 46$ & $295 \pm 48$ & $315 \pm 66$ & $279 \pm 25$ \\
\hline PWSV, mm/s & $30.4 \pm 3.4$ & $28.8 \pm 4.7$ & $20.4 \pm 5.0^{*}$ & $23.1 \pm 6.6$ \\
\hline FAC, $\%$ & $30.9 \pm 8.3$ & $35.2 \pm 6.4$ & $22.8 \pm 7.9^{*}$ & $29.7 \pm 5.5^{* * *}$ \\
\hline E-wave, $\mathrm{cm} / \mathrm{s}$ & $88 \pm 17$ & $93 \pm 28$ & $109 \pm 17$ & $103 \pm 16$ \\
\hline A wave, $\mathrm{cm} / \mathrm{s}$ & $62 \pm 26$ & $71 \pm 21$ & $38 \pm 27$ & $39 \pm 25^{* *}$ \\
\hline $\mathrm{E} / \mathrm{A}$ & $1.23 \pm 0.27$ & $1.43 \pm 0.73$ & $4.34 \pm 2.59^{*}$ & $3.95 \pm 2.54$ \\
\hline IVRT, ms & $37.0 \pm 7.8$ & $30.3 \pm 3.4$ & $30.3 \pm 4.9$ & $31.2 \pm 4.7$ \\
\hline EDT, ms & $52.7 \pm 23.9$ & $42.5 \pm 15.8$ & $36.6 \pm 8.5^{*}$ & $35.4 \pm 7.0$ \\
\hline
\end{tabular}

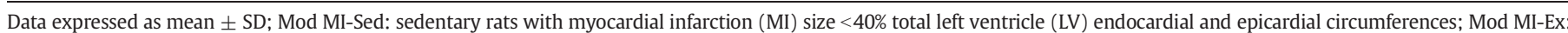

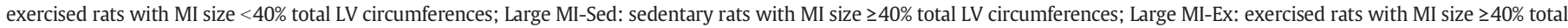

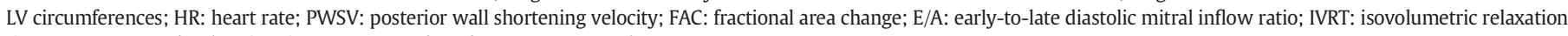
time; EDT: E-wave deceleration time. ANOVA and Student Newman-Keuls test.

* $P<0.05$ vs Mod MI-Sed.

** $P<0.05$ vs Mod MI-Ex.

*** $P<0.05$ vs Large MI-Sed. 

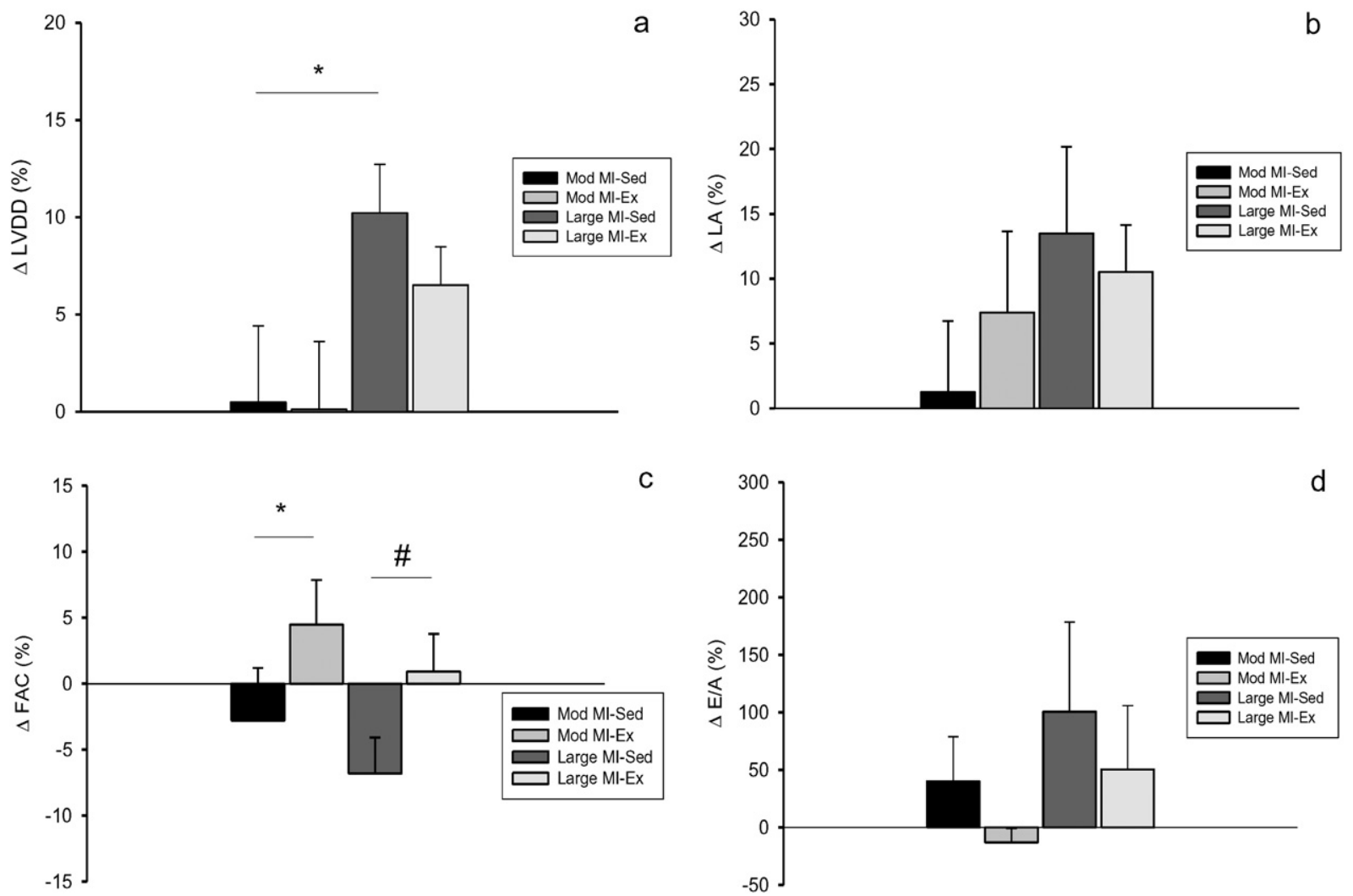

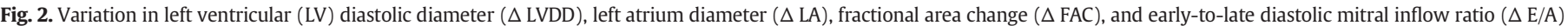

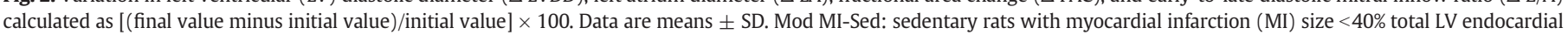

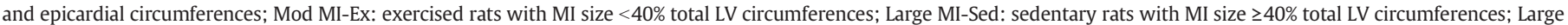
MI-Ex: exercised rats with MI size $\geq 40 \%$ total LV circumferences. ${ }^{*} P<0.05$ vs Mod MI-Sed; $\# P<0.05$ vs Large MI-Sed; ANOVA and Student Newman-Keuls test.

The effects of physical exercise on cardiac function were evaluated by echocardiogram three months after the exercise protocol. Table 3 shows cardiac structural parameters. Large MI-Sed presented higher LV diastolic diameter, left atrial diameter, and LV systolic and diastolic area than Mod IM-Sed. Large IM-Ex had a lower systolic area than large MI-Sed group. Table 4 shows LV functional data. Large MI-Sed had decreased posterior wall shortening velocity, fractional area change, and E-wave deceleration time, and higher E/A ratio than Mod MI-Sed. Large MI-Ex had higher fractional area change than Large MI-Sed and lower A wave than Mod MI-Ex group. Fig. 2 shows the percentage of variation in cardiac parameters $(\Delta)$ between final and initial periods calculated as [(final value minus initial value)/initial

Table 5

Anatomical and morphometric data.

\begin{tabular}{lllll}
\hline & $\begin{array}{l}\text { Mod MI-Sed } \\
(\mathrm{n}=7)\end{array}$ & $\begin{array}{l}\text { Mod MI-Ex } \\
(\mathrm{n}=7)\end{array}$ & $\begin{array}{l}\text { Large MI-Sed } \\
(\mathrm{n}=11)\end{array}$ & $\begin{array}{l}\text { Large MI-Ex } \\
(\mathrm{n}=10)\end{array}$ \\
\hline BW, g & $477 \pm 38$ & $482 \pm 32$ & $513 \pm 54$ & $485 \pm 32$ \\
AW, g & $0.14 \pm 0.03$ & $0.15 \pm 0.05$ & $0.28 \pm 0.13^{*}$ & $0.26 \pm 0.08^{* *}$ \\
AW/BW, mg/g & $0.30 \pm 0.08$ & $0.32 \pm 0.10$ & $0.56 \pm 0.26^{*}$ & $0.53 \pm 0.14^{* *}$ \\
RVW, g & $0.32 \pm 0.09$ & $0.34 \pm 0.11$ & $0.44 \pm 0.14^{*}$ & $0.49 \pm 0.11^{* *}$ \\
RVW/BW, mg/g & $0.68 \pm 0.26$ & $0.71 \pm 0.19$ & $0.86 \pm 0.29$ & $1.01 \pm 0.22^{* *}$ \\
LVW, g & $0.90 \pm 0.17$ & $0.96 \pm 0.14$ & $0.96 \pm 0.10$ & $0.96 \pm 0.13$ \\
LVW/BW, mg/g & $1.87 \pm 0.23$ & $2.00 \pm 0.26$ & $1.88 \pm 0.19$ & $1.98 \pm 0.23$ \\
CD, $\mu \mathrm{m}$ & $17.2 \pm 3.23$ & $16.8 \pm 4.69$ & $10.9 \pm 1.10^{*}$ & $11.5 \pm 3.00^{* *}$ \\
\hline
\end{tabular}

Data expressed as mean \pm SD. Mod MI-Sed: sedentary rats with myocardial infarction (MI) size $<40 \%$ total left ventricle (LV) endocardial and epicardial circumferences; Mod MI-Ex: exercised rats with MI size $<40 \%$ total LV circumferences; Large MI-Sed: sedentary rats with MI size $\geq 40 \%$ total LV circumferences; Large MI-Ex: exercised rats with MI size $\geq 40 \%$ total LV circumferences; BW: body weight; AW: atria weight; RVW: right ventricle weight; LVW: left ventricle weight; $\mathrm{CD}$, cardiomyocyte diameter; ANOVA and Student Newman-Keuls test.

* $P<0.05$ vs Mod MI-Sed.

** $P<0.05$ vs Mod MI-Ex. value] $\times 100$ [29]. The variation in LV diastolic diameter was higher in Large MI-Sed than Mod MI-Sed. Exercise increased $\Delta$ fractional area change in both Mod MI-Ex and Large MI-Ex compared with their respective sedentary controls.

Anatomical and morphometric data are shown in Table 5. Body weight and LV weight did not differ between groups. Both large MI groups presented higher atria and right ventricle weights than the moderate MI groups.

Myocyte $\mathrm{Ca}^{2+}$ handling protein expression is shown in Fig. 3. SERCA2a, phospholamban, and $\mathrm{Na}^{+} / \mathrm{Ca}^{2+}$ exchanger protein levels did not differ between groups. L-type calcium channel was higher in Mod MI-Ex than Mod MI-Sed and calsequestrin was higher in both exercised groups than their respective controls.

\section{Discussion}

In this study, we evaluated the effects of late physical exercise on cardiac remodeling and myocardial expression of intracellular calcium signaling proteins in rats with both moderate and large myocardial infarction.

Experimental MI has often been used for inducing heart failure. This model has the advantage of slow cardiac failure development [3], which is usually observed in clinical settings. Cardiac remodeling was characterized in this study by analyzing cardiac structures and left ventricular function with transthoracic echocardiography. Three months after MI, rats with moderate MI size presented left cardiac chambers dilation with systolic and diastolic left ventricular dysfunction compared to the Sham group. Rats with large MI had more intense left chambers dilation and left ventricular diastolic dysfunction than the Moderate MI group. At this stage, systolic function did not differ between Moderate MI and Large MI groups. Diastolic dysfunction was severe in Large MI, with a 
A

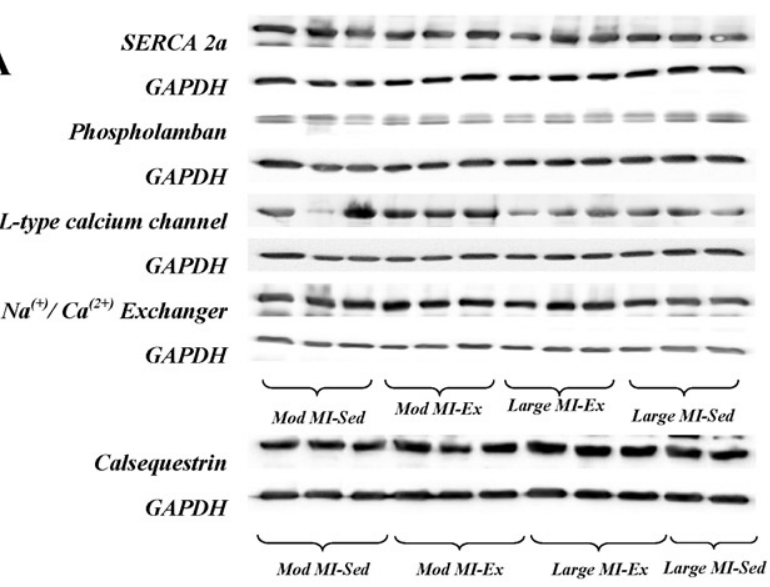

C

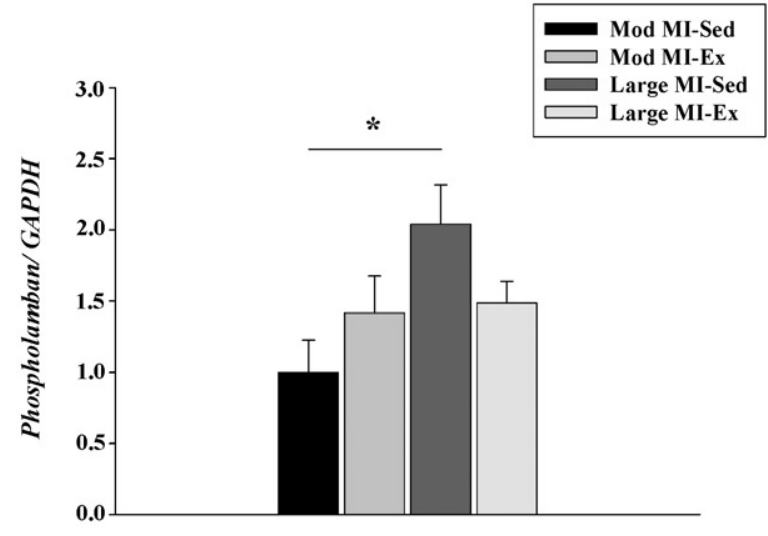

$\mathbf{E}$

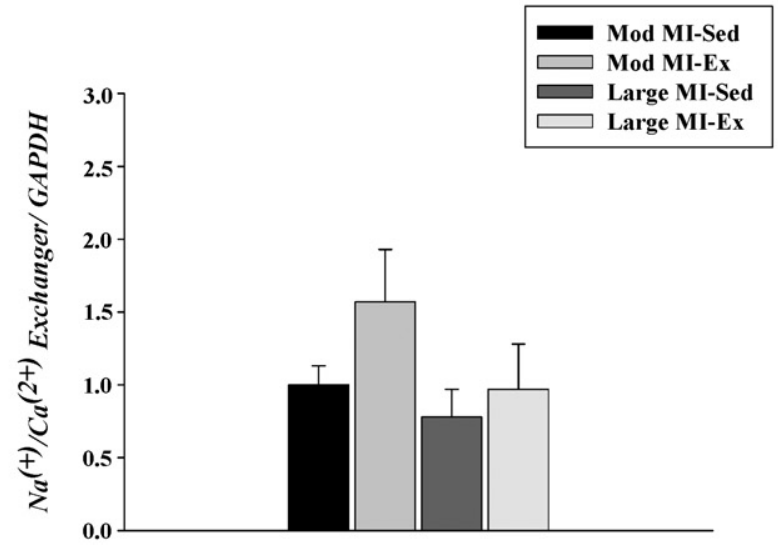

B

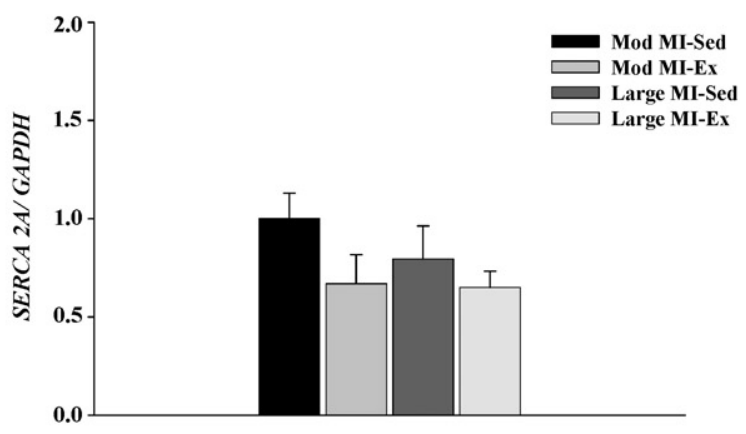

D

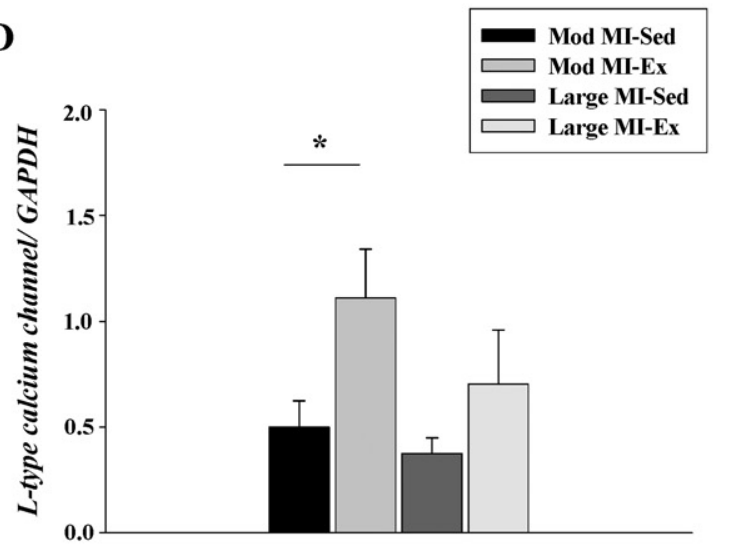

$\mathbf{F}$

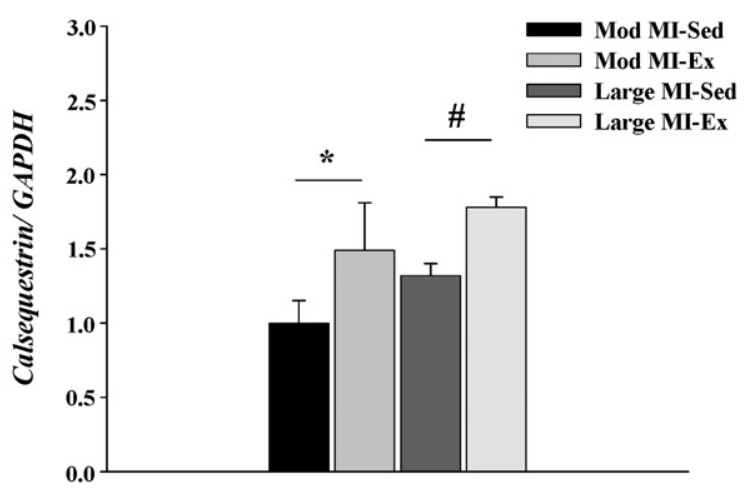

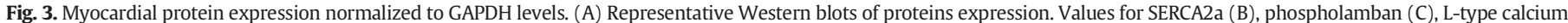

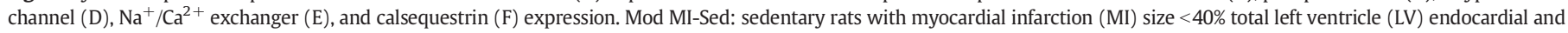

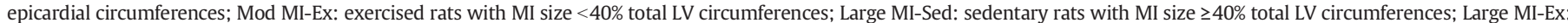

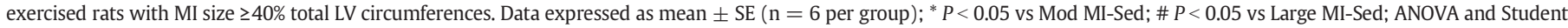
Newman-Keuls test.

restrictive pattern characterized by increased $\mathrm{E} / \mathrm{A}$ ratio and reduced E-wave deceleration time.

After the exercise period, cardiac structural and left ventricular functional echocardiographic parameters did not differ between Mod MI-Ex and Mod MI-Sed. However, variation in after and before exercise protocol fractional area change $(\triangle F A C)$ was significantly different between Mod MI-Ex and Mod MI-Sed; it was increased in Mod MI-Ex and decreased in Mod IM-Sed at the end of experiment. As left ventricular contraction asynchronism occurs in MI, fractional area change is considered the best indicator of systolic ventricular performance in this experimental model [30]. Therefore, aerobic exercise prevented systolic function impairment in rats with moderate size MI.

In large MI rats, the exercise protocol reduced systolic area and increased FAC compared to Large MI-Sed. Furthermore, $\triangle$ FAC was higher in Large MI-Ex than Large MI-Sed. Thus, exercise also prevented systolic function impairment in rats with large size infarction. Exercise did not change heart rate in moderate and large infarction groups. Heart rate was evaluated during echocardiographic evaluation with rats under a light anesthesia, which may have interfered with the measurement. 
Extrapolation of rat infarct model to clinical setting is complex. By evaluating infarct size histologically, Pfeffer et al. [31] observed that rats with infarctions less than $30 \%$ total left ventricle area do not present cardiac remodeling or ventricular dysfunction. Animals with moderate infarctions, between $30 \%$ and $45 \%$, have remodeling and dysfunction without clinical heart failure evidence. Rats with infarction size greater than $45 \%$ present remodeling, ventricular dysfunction, and heart failure features. For this reason, infarction of moderate size is considered to induce asymptomatic ventricular dysfunction, and large infarction cardiac failure.

There is substantial evidence that myocyte $\mathrm{Ca}^{2+}$ regulation abnormalities play a central role in cardiac remodeling and heart failure development in MI models [13,15,32-36]. As these mechanisms are involved in the beneficial effects of physical aerobic exercise, we evaluated myocardial protein expression related to the intracellular $\mathrm{Ca}^{2+}$ transient.

SERCA2a and phospholamban levels did not differ between groups. Exercise in moderate size MI rats increased L-type $\mathrm{Ca}^{2+}$ channel and calsequestrin expression and did not change $\mathrm{Na}^{+} / \mathrm{Ca}^{2+}$ exchanger expression. During the myocyte contraction-relaxation cycle, the sarcolemmal L-type $\mathrm{Ca}^{2+}$ channels are responsible for extracellular calcium influx into the sarcoplasm which activates calcium-induced calcium release from the sarcoplasmic reticulum [37,38]. Studies have shown decreased L-type $\mathrm{Ca}^{2+}$ channel expression in heart failure [39]. Calsequestrin is the main sarcoplasmic reticulum protein which discharges $\mathrm{Ca}^{2+}$ into the calcium release channel through its effect on the ryanodine receptor $[38,40,41]$. The role of calsequestrin in cardiac remodeling is not completely clear. Calsequestrin protein expression was reduced in infarction-induced heart failure [42]. However, massive cardiac calsequestrin overexpression (10 to 20 -fold) induced myocyte hypertrophy and dysfunction with an impaired physiological $\mathrm{Ca}^{2+}$ release mechanism [43]. It is possible that a small increase in calsequestrin in our exercised rats was involved in attenuating left ventricular dysfunction. Thus, exercise-induced improvement in systolic function was combined with increased L-type $\mathrm{Ca}^{2+}$ channel and calsequestrin expression in rats with moderate MI. As in Mod MI-Ex, the Large MI-Ex group also presented increased calsequestrin expression compared to Large MI-Sed, which can be involved in LV fractional area change improvement in Large MI-Ex rats.

In different experimental heart failure models, exercise has been shown to attenuate cardiac dysfunction $[29,44]$ and myocardial calcium handling proteins changes [37,45-47]. However, only a few authors have examined the effects of aerobic exercise after a long-term MI, which is characterized by massive structural cardiac alterations with a low probability of pharmacological or non-pharmacological reversal. In rats with large MI, Mou et al. [15] showed that late treadmill exercise attenuated systolic and myofilament dysfunction, LV dilatation, and $\mathrm{Ca}^{2+}$ transient amplitude and SERCA2a expression changes. Therefore, our study reinforces the statement that late exercise has beneficial effects on cardiac remodeling in rats with moderate and large size MI. Furthermore, to the best of our knowledge, this is the first study to show that treadmill exercise increases myocardial calsequestrin protein expression in rats with both moderate and large size MI. Additional studies are needed to establish a causal correlation between late exercise and changes in calsequestrin and the L-type calcium channel.

Our results have important clinical implication. Although current guidelines endorse cardiac rehabilitation after acute coronary syndromes, and clinical studies have shown that participation in rehabilitation after MI is associated with improvement in several outcomes, it remains unsettled whether patients with large infarction size can have the same benefits as those with moderate infarction size $[48,49]$. This study allows us to raise the hypothesis that both moderate and large MI patients can benefit from regular physical exercise.

In conclusion, late physical exercise improves systolic function and modulates intracellular calcium signaling protein expression in rats with both moderate and large myocardial infarction.

\section{Conflict of interest}

The authors report no relationship that could be construed as a conflict of interest.

\section{Ethical statement}

We adhere to the statement of ethical publishing as appears in the International Journal of Cardiology [50].

\section{Grants}

Financial support was provided by CNPq (306857/2012-0, 306845/ 2012-1, and 479085/2013-7), FAPESP (2012/21687-1 and 2010/ 02077-2), CAPES, and PROPe, UNESP.

\section{Acknowledgements}

The authors are grateful to Jose Carlos Georgette for their technical assistance and Colin Edward Knaggs for English editing.

\section{References}

[1] D. Mozaffarian, E.J. Benjamin, A.S. Go, D.K. Arnett, M.J. Blaha, M. Cushman, et al., American Heart Association Statistics Committee and Stroke Statistics Subcommittee. Heart disease and stroke statistics-2016 update: a report from the American Heart Association, Circulation 133 (2016) e38-e360.

[2] C.W. Yancy, M. Jessup, B. Bozkurt, J. Butler, D.E. Casey Jr., M.H. Drazner, et al., 2013 ACCF/AHA guideline for the management of heart failure. Executive summary: a report of the American College of Cardiology Foundation/American Heart Association task force on practice guidelines, Circulation 128 (2013) 1810-1852.

[3] P.F. Martinez, K. Okoshi, L.A. Zornoff, S.A. Oliveira Jr., D.H. Campos, A.R. Lima, et al., Echocardiographic detection of congestive heart failure in postinfarction rats, J. Appl. Physiol. 111 (2011) 543-551.

[4] M.F. Minicucci, P.S. Azevedo, P.F. Martinez, A.R. Lima, C. Bonomo, D.M. Guizoni, et al., Critical infarct size to induce ventricular remodeling, cardiac dysfunction and heart failure in rats, Int. J. Cardiol. 151 (2011) 242-243.

[5] J.C. Campos, B.B. Queliconi, P.M. Dourado, T.F. Cunha, V.O. Zambelli, L.R. Bechara, et al., Exercise training restores cardiac protein quality control in heart failure, PLoS One 7 (2012), e52764.

[6] P. Gaudron, K. Hu, R. Schamberger, M. Budin, B. Walter, G. Ertl, Effect of endurance training early or late after coronary artery occlusion on left ventricular remodeling, hemodynamics, and survival in rats with chronic transmural myocardial infarction, Circulation 89 (1994) 402-412.

[7] L. Jorge, B. Rodrigues, K.T. Rosa, C. Malfitano, T.C. Loureiro, A. Medeiros, et al., Cardiac and peripheral adjustments induced by early exercise training intervention were associated with autonomic improvement in infarcted rats: role in functional capacity and mortality, Eur. Heart J. 32 (2011) 904-912.

[8] R.B. Nunes, J.P. Alves, L.P. Kessler, P. Dal Lago, Aerobic exercise improves the inflammatory profile correlated with cardiac remodeling and function in chronic heart failure rats, Clinics 68 (2013) 876-882.

[9] L.A. Portes, R.M. Saraiva, A.A. Santos, P.J. Tucci, Swimming training attenuates remodeling, contractile dysfunction and congestive heart failure in rats with moderate and large myocardial infarctions, Clin. Exp. Pharmacol. Physiol. 36 (2009) 394-399.

[10] L.Q. Zhang, X.Q. Zhang, Y.C. Ng, L.I. Rothblum, T.I. Musch, R.L. Moore, et al., Sprint training normalizes $\mathrm{Ca}^{(2+)}$ transients and SR function in postinfarction rat myocytes, J. Appl. Physiol. 89 (2000) 38-46.

[11] A. Bansal, Q. Dai, Y.A. Chiao, K.W. Hakala, J.Q. Zhang, S.T. Weintraub, et al., Proteomic analysis reveals late exercise effects on cardiac remodeling following myocardial infarction, J. Proteome 73 (2010) 2041-2049.

[12] D.F. Batista, A.F. Gonçalves, B.P. Rafacho, P.P. Santos, M.F. Minicucci, P.S. Azevedo, et al., Delayed rather than early exercise training attenuates ventricular remodeling after myocardial infarction, Int. J. Cardiol. 170 (2013) e3-e4.

[13] U. Wisloff, J.P. Loennechen, S. Currie, G.L. Smith, O. Ellingsen, Aerobic exercise reduces cardiomyocyte hypertrophy and increases contractility, $\mathrm{Ca}^{2+}$ sensitivity and SERCA-2 in rat after myocardial infarction, Cardiovasc. Res. 54 (2002) 162-174.

[14] X. Xu, W. Wan, A.S. Powers, J. Li, L.L. Ji, S. Lao, et al., Effects of exercise training on cardiac function and myocardial remodeling in post myocardial infarction rats, J. Mol. Cell. Cardiol. 44 (2008) 114-122.

[15] Y.A. Mou, C. Reboul, L. Andre, A. Lacampagne, O. Cazorla, Late exercise training improves non-uniformity of transmural myocardial function in rats with ischaemic heart failure, Cardiovasc. Res. 81 (2009) 555-564.

[16] P.F. Martinez, C. Bonomo, D.M. Guizoni, S.A. Junior, R.L. Damatto, M.D. Cezar, et al., Influence of $N$-acetylcysteine on oxidative stress in slow-twitch soleus muscle of heart failure rats, Cell. Physiol. Biochem. 35 (2015) 148-159.

[17] A.R. Lima, P.F. Martinez, R.L. Damatto, M.D. Cezar, D.M. Guizoni, C. Bonomo, et al., Heart failure-induced diaphragm myopathy, Cell. Physiol. Biochem. 34 (2014) 333-345. 
[18] A.R. Lima, P.F. Martinez, K. Okoshi, D.M. Guizoni, L.A. Zornoff, D.H. Campos, et al. Myostatin and follistatin expression in skeletal muscles of rats with chronic heart failure, Int. J. Exp. Pathol. 91 (2010) 54-62.

[19] K. Okoshi, L.S. Matsubara, M.P. Okoshi, A.C. Cicogna, J.R. Fioretto, C.R. Padovani, et al., Food restriction-induced myocardial dysfunction demonstrated by the combination of in vivo and in vitro studies, Nutr. Res. 22 (2002) 1353-1364.

[20] S.A. Oliveira Junior, M. Dal Pai-Silva, P.F. Martinez, A.P. Lima-Leopoldo, D.H. Campos, A.S. Leopoldo, et al., Diet-induced obesity causes metabolic, endocrine and cardiac alterations in spontaneously hypertensive rats, Med. Sci. Monit. 16 (2010) BR367-BR373.

[21] R.L. Damatto, A.R. Lima, P.F. Martinez, M.D. Cezar, K. Okoshi, M.P. Okoshi, Myocardial myostatin in spontaneously hypertensive rats with heart failure, Int. J. Cardiol. 215 (2016) 384-387.

[22] M.P. Okoshi, L.S. Matsubara, M. Franco, A.C. Cicogna, B.B. Matsubara, Myocyte necrosis is the basis for fibrosis in renovascular hypertensive rats, Braz. J. Med. Biol. Res. 30 (1997) 1135-1144.

[23] P.F. Martinez, K. Okoshi, L.A. Zornoff, R.F. Carvalho, S.A. Oliveira Junior, A.R. Lima, et al., Chronic heart failure-induced skeletal muscle atrophy, necrosis, and myogenic regulatory factors changes, Med. Sci. Monit. 16 (2010) 374-383.

[24] C.M. Rosa, N.P. Xavier, D.H. Campos, A.A. Fernandes, M.D. Cezar, P.F. Martinez, et al., Diabetes mellitus activated fetal gene program and intensifies cardiac remodeling and oxidative stress in aged spontaneously hypertensive rats, Cardiovasc. Diabetol. $12(2013) 152$

[25] M.D. Cezar, R.L. Damatto, P.F. Martinez, A.R. Lima, D.H. Campos, C.M. Rosa, et al., Aldosterone blockade reduces mortality without changing cardiac remodeling in spontaneously hypertensive rats, Cell. Physiol. Biochem. 32 (2013) 1275-1287.

[26] X. Yan, A.J. Schuldt, R.L. Price, I. Amende, F.F. Liu, K. Okoshi, et al., Pressure overloadinduced hypertrophy in transgenic mice selectively overexpressing AT2 receptors in ventricular myocytes, Am. J. Physiol. Heart Circ. Physiol. 294 (2008) 1274-1281.

[27] R.L. Damatto, P.F. Martinez, A.R. Lima, M.D. Cezar, D.H. Campos, S.A.J. Oliveira, et al., Heart failure-induced skeletal myopathy in spontaneously hypertensive rats, Int. J. Cardiol. 167 (2013) 698-703.

[28] S.A. Oliveira-Junior, P.F. Martinez, D.M. Guizoni, D.H. Campos, T. Fernandes, E.M. Oliveira, et al., AT1 receptor blockade attenuates insulin resistance and myocardial remodeling in rats with diet-induced obesity, PLoS One 9 (2014), e86447.

[29] L.U. Pagan, R.L. Damatto, M.D. Cezar, A.R. Lima, C. Bonomo, D.H. Campos, et al., Long-term low intensity physical exercise attenuates heart failure development in aging spontaneously hypertensive rats, Cell. Physiol. Biochem. 36 (2015) 61-74.

[30] S.D. Solomon, S.C. Greaves, M. Ryan, P. Finn, M.A. Pfeffer, J.M. Pfeffer, Temporal dissociation of left ventricular function and remodeling following experimental myocardial infarction in rats, J. Card. Fail. 5 (1999) 213-223.

[31] M.A. Pfeffer, J.M. Pfeffer, M.C. Fishbein, P.J. Fletcher, J. Spadaro, R.A. Kloner, et al., Myocardial infarct size and ventricular function in rats, Circ. Res. 44 (1979) 503-512.

[32] U. Mackiewicz, M. Maczewski, A. Konior, J.O. Tellez, D. Nowis, H. Dobrzynski, et al., Sarcolemmal $\mathrm{Ca}^{2+}$-ATPase ability to transport $\mathrm{Ca}^{2+}$ gradually diminishes after myocardial infarction in the rat, Cardiovasc. Res. 81 (2009) 546-554.

[33] Q. Shao, B. Ren, A. Zarain-Herzberg, P.K. Ganguly, N.S. Dhalla, Captopril treatment improves the sarcoplasmic reticular $\mathrm{Ca}^{(2+)}$ transport in heart failure due to myocardial infarction, J. Mol. Cell. Cardiol. 31 (1999) 1663-1672.
[34] A.G. Brittsan, E.G. Kranias, Phospholamban and cardiac contractile function, J. Mol. Cell. Cardiol. 32 (2000) 2131-2139.

[35] K. Ito, X. Yan, X. Feng, W.J. Manning, W.H. Dillmann, B.H. Lorell, Transgenic expression of sarcoplasmic reticulum $\mathrm{Ca}^{(2+)}$ ATPase modifies the transition from hypertrophy to early heart failure, Circ. Res. 89 (2001) 422-429.

[36] A.L. Gut, M.P. Okoshi, C.R. Padovani, F.F. Aragon, A.C. Cicogna, Myocardial dysfunction induced by food restriction is related to calcium cycling and beta-adrenergic system changes, Nutr. Res. 23 (2003) 911-919.

[37] J. Locatelli, L.V. de Assis, M.C. Isoldi, Calcium handling proteins: structure, function, and modulation by exercise, Heart Fail. Rev. 19 (2014) 207-225.

[38] L.H. Opie, G. Hasenfuss, Mechanisms of cardiac contraction and relaxation, Braunwald's Heart Disease. A Textbook of Cardiovascular Medicine, Elsevier Saunders, Philadelphia 2012, pp. 459-486.

[39] S.R. Houser, K.B. Margulies, Is depressed myocyte contractility centrally involved in heart failure? Circ. Res. 92 (2003) 350-358.

[40] Z. Kubalova, I. Gyorke, R. Terentyeva, S. Viatchenko-Karpinski, D. Terentyev, S.C. Williams, et al., Modulation of cytosolic and intra-sarcoplasmic reticulum calcium waves by calsequestrin in rat cardiac myocytes, J. Physiol. 561 (2004) 515-524.

[41] S. Jacob, N.H. Sleiman, S. Kern, L.R. Jones, J.A. Sala-Mercado, T.P. McFarland, et al., Altered calsequestrin glycan processing is common to diverse models of canine heart failure, Mol. Cell. Biochem. 377 (2013) 11-21.

[42] S.T. Hu, G.S. Liu, Y.F. Shen, Y.L. Wang, Y. Tang, Y.J. Yang, Defective $\mathrm{Ca}^{(2+)}$ handling proteins regulation during heart failure, Physiol. Res. 60 (2011) 27-37.

[43] L.R. Jones, Y.J. Suzuki, W. Wang, Y.M. Kobayashi, V. Ramesh, C. Franzini-Armstrong et al., Regulation of $\mathrm{Ca}^{2+}$ signaling in transgenic mouse cardiac myocytes overexpressing calsequestrin, J. Clin. Invest. 101 (1998) 1385-1393.

[44] M.C. de Waard, J. van der Velden, V. Bito, S. Ozdemir, L. Biesmans, N.M. Boontje et al., Early exercise training normalizes myofilament function and attenuates left ventricular pump dysfunction in mice with a large myocardial infarction, Circ. Res. 100 (2007) 1079-1088

[45] L. Lu, D.F. Mei, A.G. Gu, S. Wang, B. Lentzner, D.E. Gutstein, et al., Exercise training normalizes altered calcium-handling proteins during development of heart failure, J. Appl. Physiol. 92 (2002) 1524-1530.

[46] A. Medeiros, N.P. Rolim, R.S. Oliveira, K.T. Rosa, K.C. Mattos, D.E. Casarini, et al., Exercise training delays cardiac dysfunction and prevents calcium handling abnormalities in sympathetic hyperactivity-induced heart failure mice, J. Appl. Physiol. 104 (2008) 103-109.

[47] P.M. Buttrick, M. Kaplan, L.A. Leinwand, J. Scheuer, Alterations in gene expression in the rat heart after chronic pathological and physiological loads, J. Mol. Cell. Cardiol. 26 (1994) 61-67.

[48] K.G. Aragam, D. Dai, M.L. Neely, D.L. Bhatt, M.T. Roe, J.S. Rumsfeld, et al., Gaps in referral to cardiac rehabilitation of patients undergoing percutaneous coronary intervention in the United States, J. Am. Coll. Cardiol. 65 (2015) 2079-2088.

[49] B.S. Heran, J.M. Chen, S. Ebrahim, T. Moxham, N. Oldridge, K. Rees, et al., Exercisebased cardiac rehabilitation for coronary heart disease, Cochrane Database Syst. Rev. 7 (2011), CD001800.

[50] L.G. Shewan, G.M.C. Rosano, M.Y. Henein, A.J.S. Coats, A statement on ethical standards in publishing scientific articles in the International Journal of Cardiology family of journals, Int. J. Cardiol. 170 (2014) 253-254. 